\title{
GLOBAL COSMIC-RAY-RELATED LUMINOSITY AND ENERGY BUDGET OF THE MILKY WAY
}

\author{
A. W. Strong ${ }^{1}$, T. A. Porter ${ }^{2}$, S. W. Digel ${ }^{3,4}$, G. Jóhannesson ${ }^{2}$, P. Martin $^{1}$, I. V. Moskalenko ${ }^{2,4}$, E. J. Murphy $^{5}$, \\ AND E. ORLANDO 1 \\ ${ }^{1}$ Max-Planck-Institut für extraterrestrische Physik, Postfach 1312, D-85741 Garching, Germany; aws@ mpe.mpg.de \\ ${ }^{2}$ Hansen Experimental Physics Laboratory, Stanford University, Stanford, CA 94305, USA; tporter@ stanford.edu \\ ${ }^{3}$ SLAC National Accelerator Laboratory, 2575 Sand Hill Road, Menlo Park, CA 94025, USA; digel@ slac.stanford.edu \\ ${ }^{4}$ Kavli Institute for Particle Astrophysics and Cosmology, Stanford University, Stanford, CA 94305, USA \\ ${ }^{5}$ Spitzer Science Center, California Institute of Technology, Pasadena, CA 91125, USA; emurphy@ipac.caltech.edu \\ Received 2010 June 14; accepted 2010 August 24; published 2010 September 20
}

\begin{abstract}
We use the GALPROP code for cosmic-ray (CR) propagation to calculate the broadband luminosity spectrum of the Milky Way related to CR propagation and interactions in the interstellar medium. This includes $\gamma$-ray emission from the production and subsequent decay of neutral pions $\left(\pi^{0}\right)$, bremsstrahlung, and inverse Compton scattering, and synchrotron radiation. The Galaxy is found to be nearly a CR electron calorimeter, but only if $\gamma$-ray emitting processes are taken into account. Synchrotron radiation alone accounts for only one-third of the total electron energy losses with $\sim 10 \%-20 \%$ of the total synchrotron emission from secondary CR electrons and positrons. The relationship between far-infrared and radio luminosity that we find from our models is consistent with that found for galaxies in general. The results will be useful for understanding the connection between diffuse emissions from radio through $\gamma$-rays in "normal" (non-active galactic nucleus dominated) galaxies as well as for estimating the broadband extragalactic diffuse background from these kinds of galaxies.
\end{abstract}

Key words: cosmic rays - Galaxy: general - gamma rays: galaxies - gamma rays: general - radiation mechanisms: non-thermal - radio continuum: galaxies

Online-only material: color figures

\section{INTRODUCTION}

Cosmic rays (CRs) fill up the entire volume of galaxies, providing an important source of heating and ionization of the interstellar medium (ISM), and may play a significant role in the regulation of star formation and evolution of galaxies (Ferrière 2001; Cox 2005; Socrates et al. 2008; Sironi \& Socrates 2010). Diffuse emissions from radio to high-energy $\gamma$-rays $(>100 \mathrm{MeV})$ arising from various interactions between CRs and the ISM, interstellar radiation field (ISRF), and magnetic field, are currently the best way to trace the intensities and spectra of CRs in the Milky Way (MW) and other galaxies. Gamma rays are particularly useful in this respect since this energy range gives access to the dominant hadronic component in CRs via the observation of $\pi^{0}$-decay radiation produced by CR nuclei inelastically colliding with the interstellar gas. Understanding the global energy budget of processes related to the injection and propagation of CRs, and how the energy is distributed across the electromagnetic spectrum, is essential to interpreting the radio/farinfrared relation (de Jong et al. 1985; Helou et al. 1985; Murphy et al. 2006), galactic calorimetry (e.g., Völk 1989), and predictions of extragalactic backgrounds (e.g., Thompson et al. 2007; Murphy et al. 2008), and for many other studies.

The MW is the best studied non-active galactic nucleus dominated star-forming galaxy, and the only galaxy that direct measurements of $\mathrm{CR}$ intensities and spectra are available. However, because of our position inside, the derivation of global properties is not straightforward and requires detailed models of the spatial distribution of the emission. Nevertheless, constructing a model for the global properties of such a galaxy is tractable with the variety of data available.

In this Letter, we calculate the injected CR power and corresponding broadband luminosity spectrum from radio to $\gamma$-rays for CR propagation models consistent with current CR, radio, and $\gamma$-ray data. Earlier estimates focusing on the MW $\gamma$-ray luminosity only (e.g., Strong et al. 2000) were based on modeling EGRET data. The launch of the Large Area Telescope (LAT) on the Fermi Gamma-ray Space Telescope (hereafter Fermi-LAT) has provided a wealth of new $\gamma$-ray data up to, and beyond, $100 \mathrm{GeV}$. Analysis of the Fermi-LAT data has not confirmed the anomalous "EGRET GeV-excess" (Abdo et al. 2009a, 2009c) and has led to an improved model of CR propagation and diffuse $\gamma$-ray emission thus enabling a better estimate of the CR and $\gamma$-ray luminosities of the Galaxy.

\section{THE MODELS}

We use a model based on the GALPROP $\operatorname{code}^{6}$ for the CR-related processes in the Galaxy that has been adjusted to fit many types of data including direct measurements of CRs, non-thermal radio emission, hard X-rays, and $\gamma$-rays. Studies of the diffuse Galactic emission and CRs using this code prior to the launch of the Fermi-LAT can be found in Moskalenko \& Strong (1998), Strong \& Moskalenko (1998), Strong et al. (2000, 2004a, 2004b), Moskalenko et al. (2002), and Porter et al. (2008). An extensive review of CR propagation, models, data, and literature is given by Strong et al. (2007).

We calculate diffuse emissions from radio to $\gamma$-rays, produced by $\mathrm{CR}$ protons, helium, and electrons/positrons. The contributions by discrete sources and line emissions (e.g., $511 \mathrm{keV}$ annihilation radiation) are not included. Since the propagation parameters are not uniquely determined from the observations, we consider an illustrative range of parameters for diffusive reacceleration (DR ) and plain diffusion (PD) propagation models. The main uncertainty we consider is the CR confinement

\footnotetext{
6 http://galprop.stanford.edu
} 
Table 1

GALPROP Model Parameters

\begin{tabular}{|c|c|c|c|c|c|c|}
\hline \multirow[t]{2}{*}{ Podel Parameter/GALDEF ID } & \multicolumn{3}{|c|}{ Diffusive Reacceleration } & \multicolumn{3}{|c|}{ Plain Diffusion } \\
\hline & $\begin{array}{l}\text { Model } 1 \\
\text { z02LMS }\end{array}$ & $\begin{array}{l}\text { Model } 2 \\
\text { z04LMS }\end{array}$ & $\begin{array}{l}\text { Model } 3 \\
\text { z10LMS }\end{array}$ & $\begin{array}{c}\text { Model } 1 \\
\text { z02LMPDS }\end{array}$ & $\begin{array}{c}\text { Model } 2 \\
\text { z04LMPDS }\end{array}$ & $\begin{array}{c}\text { Model } 3 \\
\text { z10LMPDS }\end{array}$ \\
\hline Halo height, kpc & 2 & 4 & 10 & 2 & 4 & 10 \\
\hline Galaxy radius, $\mathrm{kpc}$ & 20 & 20 & 20 & 20 & 20 & 20 \\
\hline Diffusion coefficient ${ }^{\text {a }} D_{0}$ & 2.9 & 5.8 & 10.0 & 1.8 & 3.4 & 6.0 \\
\hline Diffusion coefficient $^{\mathrm{a}} \delta$ & 0.33 & 0.33 & 0.33 & 0.5 & 0.5 & 0.5 \\
\hline Reacceleration $v_{A}, \mathrm{~km} \mathrm{~s}^{-1}$ & 30 & 30 & 30 & $\ldots$ & $\ldots$ & $\ldots$ \\
\hline $\begin{array}{l}\text { CR sources distribution } \\
\text { Magnetic field strength }^{\mathrm{c}}\end{array}$ & \multicolumn{6}{|c|}{$\begin{array}{c}\text { Pulsars (Lorimer 2004) } \\
B=7 e^{-\left(R-R_{0}\right) / R_{B}-z / z_{B}} \mu \mathrm{G}\end{array}$} \\
\hline \multicolumn{7}{|l|}{ Injection spectrum (nuclei) } \\
\hline Index below break & 1.98 & 1.98 & 1.98 & 1.80 & 1.80 & 1.80 \\
\hline Index above break & 2.42 & 2.42 & 2.42 & 2.25 & 2.25 & 2.25 \\
\hline Break energy, GeV & 9 & 9 & 9 & 9 & 9 & 9 \\
\hline Normalization energy, $\mathrm{GeV}$ & 100 & 100 & 100 & 100 & 100 & 100 \\
\hline Proton normalization intensity ${ }^{\mathrm{d}, \mathrm{e}}$ & 5 & 5 & 5 & 5 & 5 & 5 \\
\hline \multicolumn{7}{|l|}{ Injection spectrum (primary electrons) } \\
\hline Index below break & 1.60 & 1.60 & 1.60 & 1.80 & 1.80 & 1.80 \\
\hline Index above break & 2.42 & 2.42 & 2.42 & 2.25 & 2.25 & 2.25 \\
\hline Break energy, GeV & 4 & 4 & 4 & 4 & 4 & 4 \\
\hline Normalization energy, $\mathrm{GeV}$ & 34.5 & 34.5 & 34.5 & 34.5 & 34.5 & 34.5 \\
\hline Normalization intensity ${ }^{\mathrm{d}}$ & 0.32 & 0.32 & 0.32 & 0.32 & 0.32 & 0.32 \\
\hline
\end{tabular}

Notes.

${ }^{\text {a }} D_{x x}=10^{28} \beta D_{0}\left(\rho / \rho_{0}\right)^{\delta} \mathrm{cm}^{2} \mathrm{~s}^{-1}, \rho_{0}=4 \mathrm{GV}, \beta=v / c$, constant below $\rho_{0}$ for PD model.

${ }^{\mathrm{b}}$ Modified to have the value at $R=10 \mathrm{kpc}$ across the range $R=10-15 \mathrm{kpc}$ and to be zero beyond $15 \mathrm{kpc}$.

${ }^{\mathrm{c}} R_{0}=8.5 \mathrm{kpc}, R_{B}=50 \mathrm{kpc}, z_{B}=3 \mathrm{kpc}$.

d $10^{-9} \mathrm{~cm}^{-2} \mathrm{~s}^{-1} \mathrm{sr}^{-1} \mathrm{MeV}^{-1}$ (nucleon ${ }^{-1}$ for nuclei).

${ }^{\mathrm{e}}$ Normalization energy $100 \mathrm{GeV}$ for protons corresponds to a rigidity $\sim 100 \mathrm{GV}$. Helium normalization is 0.068 relative to protons at the same rigidity.

volume and associated propagation model parameters, e.g., the diffusion coefficient. Other effects to consider would include different propagation modes, e.g., convection, the distribution of CR sources, etc. However, these are beyond the scope of the current Letter and we defer these considerations to future work. The output of a typical GALPROP run includes CR distributions, $\gamma$-ray/synchrotron sky maps, and emissivity distributions. The luminosities of the different components are computed by integrating the respective emissivities over the total Galactic volume: for inverse Compton (IC) and synchrotron radiation the volume emissivity is directly calculated, while for $\pi^{0}$-decay and bremsstrahlung the emissivity is calculated per hydrogen atom, so we weight the calculated volume emissivities by the distributions of atomic, molecular, and ionized hydrogen included in the GALPROP code (Strong \& Moskalenko 1998; Moskalenko et al. 2002; Strong et al. 2004b).

We obtain the input CR luminosity for each of our models by integrating the model CR source spectra and spatial distribution over the Galaxy. The injected luminosity, instead of emergent, is the relevant quantity because we are interested in how the CR power is transferred to the electromagnetic channels. In addition to $\mathrm{CR}$ protons and helium, and primary electrons, we also calculate the luminosities of secondary positrons and electrons resulting from CR-gas interactions in the ISM. The diffuse emissions from these secondary CR species are also calculated and included in the total synchrotron, bremsstrahlung, and IC spectra.

We use DR and PD propagation models where the spatial diffusion coefficient $D_{x x}$ and its momentum dependence, together with the DR characterized by an Alfvén speed, $v_{A}$, assuming a Kolmogorov spectrum of interstellar turbulence (if used), and the size of the CR confinement volume, $z_{h}$, are obtained by fitting the $\mathrm{CR}$ secondary/primary ratios for $\mathrm{B} / \mathrm{C}$ and ${ }^{10} \mathrm{Be} /{ }^{9} \mathrm{Be}$; for details see Strong et al. (2007). The CR source injection spectra are taken as broken power laws in momentum, with different parameters for nuclei and primary electrons. These are chosen to reproduce the directly observed CR spectra after propagation for the adopted models.

The calculations are based on model parameters that reproduce the Fermi-LAT electron spectrum (Abdo et al. 2009a) and $\gamma$-ray data (Abdo et al. 2009a, 2009b, 2010a, 2010b). The spatial distribution of the CR sources is based on pulsars as tracers of supernova remnants (SNRs) used in Strong et al. (2004b), but constant beyond the solar circle as indicated by analysis of the Fermi-LAT data for the 2nd Galactic quadrant (Abdo et al. 2010a).

The synchrotron calculation uses the same CR electron model and a magnetic field model that reproduces radio surveys at frequencies from $100 \mathrm{MHz}$ to $23 \mathrm{GHz}$ (A. W. Strong et al. 2011 , in preparation). DR and PD propagation models are used for three halo sizes, $z_{h}=2,4$, and $10 \mathrm{kpc}$, respectively, with corresponding self-consistently derived diffusion coefficients $D_{x x}(\rho)=D_{0} \beta\left(\rho / \rho_{0}\right)^{\delta}$, where $\beta=v / c$ is the dimensionless particle velocity, $\rho$ is the particle rigidity, and $D_{0}, \rho_{0}, \delta$ are constants. These halo sizes cover the range consistent with available CR data for B/C and ${ }^{10} \mathrm{Be} /{ }^{9} \mathrm{Be}$ (Engelmann et al. 1990; Yanasak et al. 2001; de Nolfo et al. 2006, and references therein). The parameters and values for the models are summarized in Table 1.

The optical to far-infrared (FIR) luminosity spectrum is derived from the model of the ISRF used for the propagation calculations (the "maximum metallicity gradient" model from 

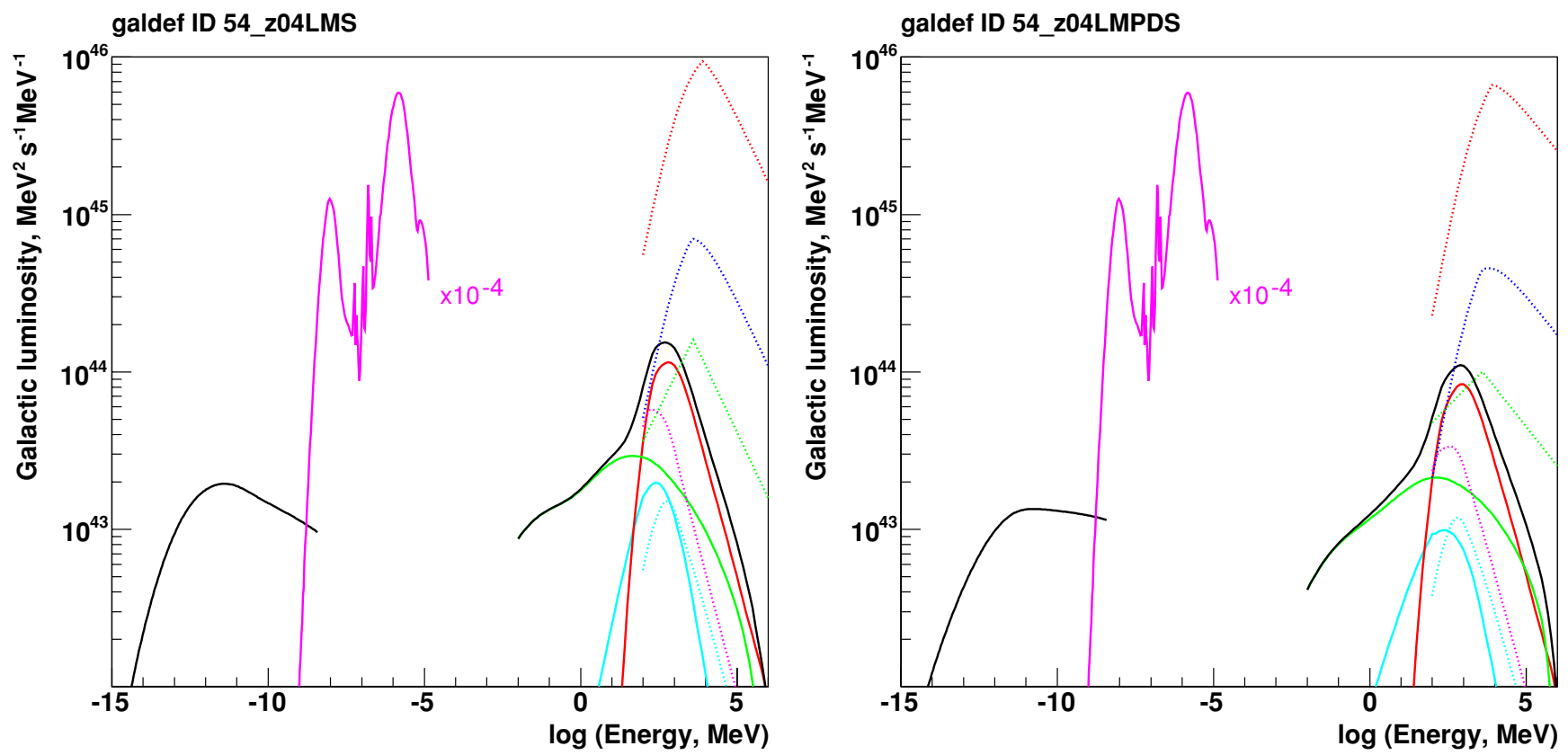

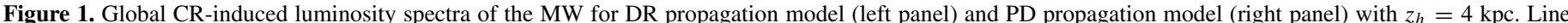

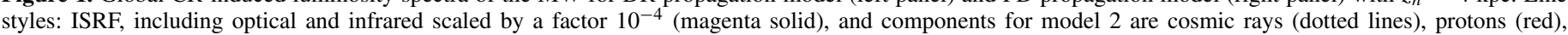

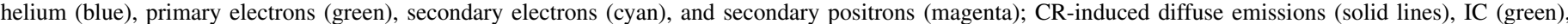
bremsstrahlung (cyan), $\pi^{0}$-decay (red), synchrotron (black, the left side of figure), and total (black, the right side of figure).

(A color version of this figure is available in the online journal.)

Porter et al. 2008). The emergent luminosity for the ISRF is computed by surface integration over a region large enough to encompass the total flux from the stellar luminosity distribution and the starlight reprocessed by dust $(\sim 30 \mathrm{kpc}$ radius about the Galactic center). In the present work, the input bolometric stellar luminosity is $\sim 4 \times 10^{10} L_{\odot}$ apportioned across the stellar components boxy bulge/thin disc/thick disc/halo with fractions $\sim 0.1 / 0.7 / 0.1 / 0.1$, and $\sim 20 \%$ reprocessed by dust and emitted in the infrared. The uncertainties related to the distribution of the ISRF interior to the integration boundary include the relative luminosities of the bulge component and the disk, the metallicity gradient, and other details. However, these uncertainties are dominated by the overall uncertainty in the input stellar luminosity. A higher input stellar luminosity will increase the CR electron/positron losses via IC scattering and hence the overall output in $\gamma$-rays, requiring a higher injected $\mathrm{CR}$ power and increasing the overall calorimetric efficiency (see below). Estimates available in the literature illustrating the range for the MW stellar luminosity are, e.g., $6.7 \times 10^{10} L_{\odot}$ (Kent et al. 1991) and $2.3 \times 10^{10} L_{\odot}$ (Freudenreich 1998).

\section{RESULTS AND DISCUSSION}

Figure 1 shows the broadband luminosity spectrum of the Galaxy, including the input luminosity for CRs for a $4 \mathrm{kpc}$ halo for a DR and PD model, respectively. ${ }^{7}$ Figure 2 illustrates the detailed energy budget for the DR model with $4 \mathrm{kpc}$ halo size. Figure 3 shows the broadband luminosities for DR and PD models for the three halo sizes, and Table 2 summarizes the spectrally integrated luminosity for the various components for each of our models. The peak injected CR luminosities differ by a factor $\sim 2$ for different propagation modes, but the total injected CR luminosities are close, only differing at the $\sim$ few

\footnotetext{
7 Spectra for all models and components will be made available in numerical form via the German Virtual Observatory, http://www.g-vo.org.
}

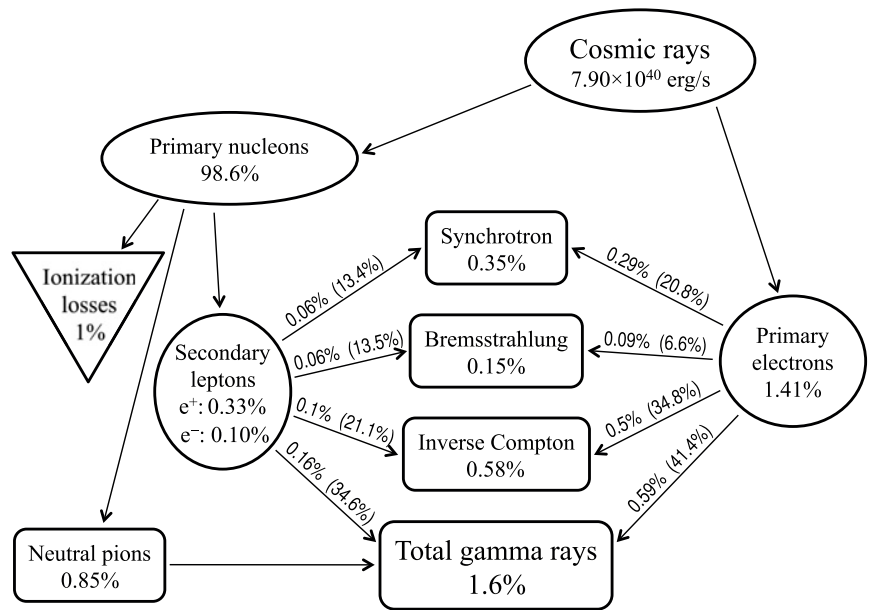

Figure 2. Luminosity budget of the MW for DR propagation model with $z_{h}=4 \mathrm{kpc}$. The percentage figures are shown with respect to the total injected luminosity in CRs, $7.9 \times 10^{40} \mathrm{erg} \mathrm{s}^{-1}$. The percentages in brackets show the values relative to the luminosity of their respective lepton populations (primary electrons, secondary electrons/positrons).

percent level. The injected CR spectra differ between DR and PD models to compensate the momentum dependence of the diffusion coefficient in each propagation model so that the local $\mathrm{CR}$ spectra are reproduced. Independent of propagation mode, the relative decrease in the injected $\mathrm{CR}$ proton and helium luminosities is $\sim 10 \%$ for halo sizes $2-10 \mathrm{kpc}$. For smaller halo sizes, the CRs escape quicker requiring more injected power to maintain the local CR spectrum. In addition, for larger halo sizes CR sources located at further distances can contribute to the local spectrum, which is our normalization condition, hence less power is required. In contrast to nuclei, the injected primary CR electron luminosity increases with $z_{h}$, reflecting the increased input power of these particles required to counter the 

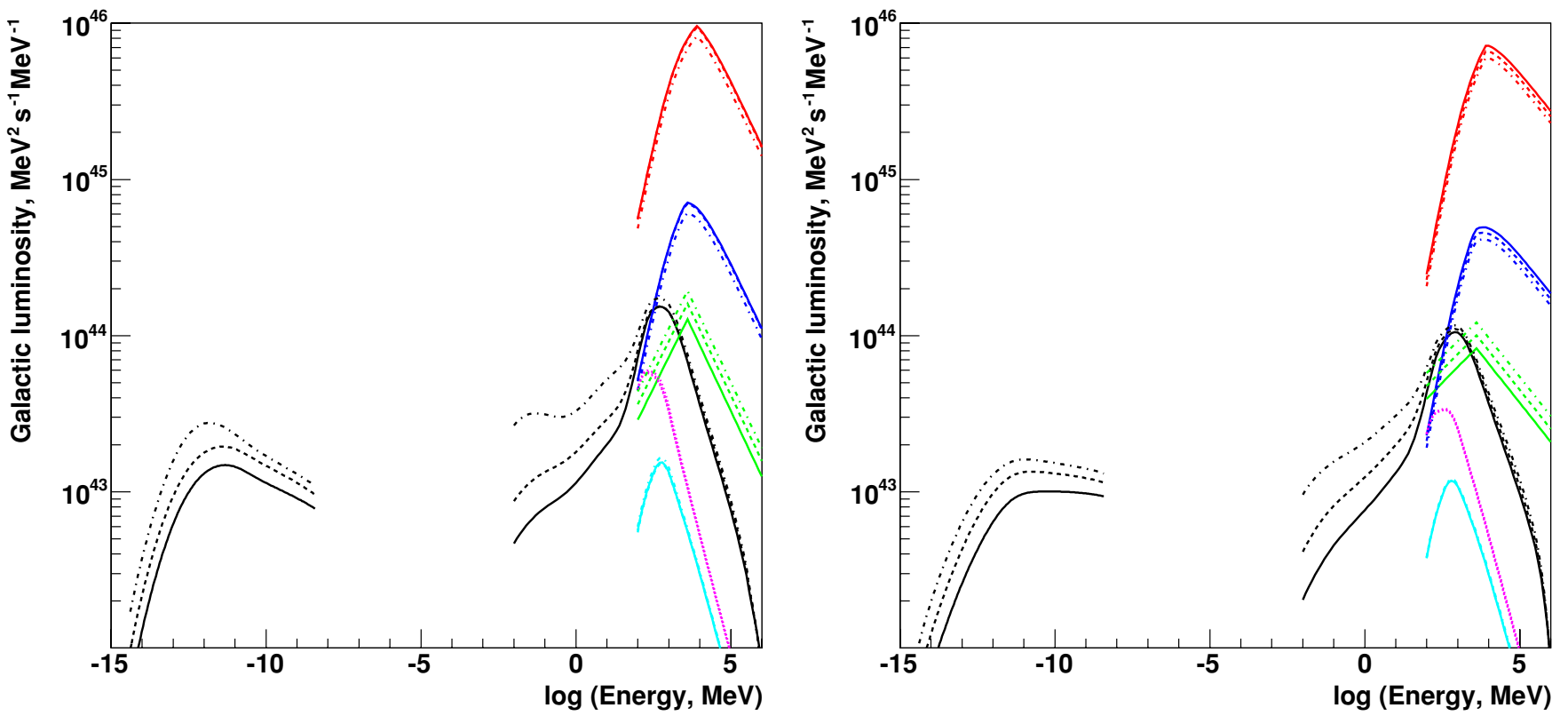

Figure 3. Global CR-induced luminosity spectra of the MW for a range of halo sizes for DR propagation model (left panel) and PD propagation model (right panel). Line styles: $z_{h}=2 \mathrm{kpc}$ (solid), $z_{h}=4 \mathrm{kpc}$ (dotted), and $z_{h}=10 \mathrm{kpc}$ (dot-dashed). Components as in Figure 1.

(A color version of this figure is available in the online journal.)

energy losses from the larger confinement region and escape time.

The CR nuclei luminosities can be directly compared with the well-known approximate estimate from CR "grammage" as described by, e.g., Dogiel et al. (2002), which shows that these can be estimated from the observed secondary/primary ratios. The values in Table 2 are within a factor of 2 of the $\sim 10^{41} \mathrm{erg} \mathrm{s}^{-1}$ derived in that paper, providing a consistency check on our more detailed modeling. Taking the CR luminosities and adopting a supernova rate of (1.9 \pm 1.1$)$ /century (Diehl et al. 2006), we obtain a CR energy input per SNR of $(0.3-1) \times$ $10^{50} \mathrm{erg}$, comparable to standard estimates of a 5\%-10\% efficiency for CR acceleration in SNRs.

For the DR model, the injected CR lepton luminosity is close to the total of synchrotron, IC, and bremsstrahlung luminosities for the $10 \mathrm{kpc}$ halo (Model 3), showing that the Galaxy is approximately a lepton calorimeter ( $\sim 79 \%$ efficiency) in this case, with the bulk of the energy lost via IC emission (only about one-third is lost via synchrotron emission). Smaller halos still constitute fair calorimeters but are less efficient: $\sim 51 \%$ for the case of a $2 \mathrm{kpc}$ halo with $\sim 17 \%$ of the energy loss due to synchrotron radiation, and $\sim 59 \%$ for the case of a $4 \mathrm{kpc}$ halo with $\sim 19 \%$ of the energy loss due to synchrotron radiation. For PD models the calorimetric efficiencies are decreased, but the same trend of higher efficiency with increasing halo size is evident.

Our calculations show that calorimetry holds for CR leptons in MW-like galaxies independent of propagation mode provided the size of the CR confinement volume is large enough to allow for sufficient cooling and if $\gamma$-ray production is included. A large fraction of the IC luminosity is at energies below $100 \mathrm{MeV}$, extending to hard X-rays, and includes a significant secondary electron/positron component (Strong et al. 2004a; Porter et al. 2008). The contribution to the synchrotron emission from secondary electrons and positrons is $\sim 10 \%-20 \%$ for our Galaxylike models, being largest for small halo sizes. For DR models, the secondary lepton emission is $20 \%, 16 \%$, and $16 \%$ for 2, 4, and $10 \mathrm{kpc}$, respectively. For PD models, the secondary lepton emission is $12 \%, 10 \%$, and $10 \%$ for 2 , 4 , and $10 \mathrm{kpc}$, respectively.

Using the standard definition of the FIR/radio relation, $q=\log _{10}\left(S_{\mathrm{FIR}} / 3.75 \times 10^{12} \mathrm{~Hz}\right)-\log _{10}\left(S_{1.4 \mathrm{GHz}}\right)$, where $S_{\mathrm{FIR}}$ (W m ${ }^{-2}$ ) is the FIR flux from 42.5 to $122.5 \mu \mathrm{m}$ and $S_{1.4 \mathrm{GHz}}$ ( $\mathrm{W} \mathrm{m}^{-2} \mathrm{~Hz}^{-1}$ ) is the radio flux at $1.4 \mathrm{GHz}$ (Helou et al. 1985), we calculate this ratio for our luminosity models (see Table 2 under "radio-FIR relation"). The range of values $2.26-2.69$ is consistent with that for the correlation over normal and starburst galaxies: $2.34 \pm 0.26$ (Yun et al. 2001) and near to the value for M33 (2.50) given in that paper. Note, here we have used the FIR luminosity from the ISRF model described earlier, $1.9 \times$ $10^{43} \mathrm{erg} \mathrm{s}^{-1}$ (see Table 2). However, the FIR luminosity of the Galaxy is uncertain by at least a factor of 2 and this will affect $q$. Using COBE/DIRBE data, Sodroski et al. (1997) give a total IR luminosity $4.1 \times 10^{43} \mathrm{erg} \mathrm{s}^{-1}$ and IR luminosity $>40 \mu \mathrm{m}$, $\mathrm{IR}_{>40 \mu \mathrm{m}}$, of $2.8 \times 10^{43} \mathrm{erg} \mathrm{s}^{-1}$. This yields an FIR luminosity of $1.4 \times 10^{43} \mathrm{erg} \mathrm{s}^{-1}$ from FIR/IR $>40 \mu \mathrm{m}=0.51$ for the ISRF used in this Letter. Meanwhile, Paladini et al. (2007) give a much higher value for $\mathrm{IR}_{>60 \mu \mathrm{m}}, 1.1 \times 10^{44} \mathrm{erg}^{-1}$, yielding an FIR luminosity $6.6 \times 10^{43} \mathrm{erg} \mathrm{s}^{-1}$ (R. Paladini 2010, private communication). This higher value for the FIR luminosity would increase all $q$ values in Table 2 by 0.53 .

The luminosity spectra for our models contain significant IC $\gamma$-ray emission, the contribution at high energies depending on details of the propagation model. Above $\sim 10 \mathrm{GeV}$ the contribution by IC is non-negligible compared to $\pi^{0}$-decay. Estimates of the isotropic background using models for the diffuse $\gamma$-ray emission of normal star-forming galaxies invoking only $\pi^{0}$-decay as the dominant mode for high-energy production, e.g., Ando \& Pavlidou (2009), and ignoring details of the propagation will underestimate the contribution per galaxy by these classes of objects.

The present estimates are intended to be illustrative, using a particular set of models; the level of uncertainty is suggested by the range covered by the models, which are far from exhaustive. In any case the general conclusions from this work are robust. 
Table 2

Luminosity of the Galaxy for Various Processes, $10^{38} \mathrm{erg} \mathrm{s}^{-1}$

\begin{tabular}{|c|c|c|c|c|c|c|}
\hline \multirow[t]{2}{*}{ Component } & \multicolumn{3}{|c|}{ Diffusive Reacceleration } & \multicolumn{3}{|c|}{ Plain Diffusion } \\
\hline & Model 1 & Model 2 & Model 3 & Model 1 & Model 2 & Model 3 \\
\hline Cosmic rays $(0.1-100 \mathrm{GeV})$ : & 805 & 790 & 698 & 780 & 723 & 660 \\
\hline Protons & 737 & 724 & 633 & 718 & 662 & 601 \\
\hline Helium & 56 & 55 & 48 & 52.4 & 48.3 & 43.9 \\
\hline Leptons & 12.2 & 14.5 & 16.9 & 10.04 & 12.91 & 15.15 \\
\hline Primary $e^{-}$ & 8.8 & 11.1 & 13.4 & 8.65 & 10.5 & 12.7 \\
\hline Secondary $e^{-}$ & 0.78 & 0.77 & 0.83 & 0.63 & 0.64 & 0.65 \\
\hline Secondary $e^{+}$ & 2.6 & 2.6 & 2.7 & 1.76 & 1.77 & 1.80 \\
\hline$\gamma$-rays $(0.01-100 \mathrm{MeV})$ & 2.32 & 3.34 & 6.22 & 1.47 & 2.20 & 3.50 \\
\hline$\pi^{0}$-decay & 0.24 & 0.23 & 0.23 & 0.14 & 0.13 & 0.13 \\
\hline Inverse Compton & 1.80 & 2.81 & 5.63 & 1.09 & 1.79 & 3.04 \\
\hline Primary $e^{-}$ & 1.31 & 2.20 & 4.41 & 0.91 & 1.54 & 2.67 \\
\hline Secondary $e^{ \pm}$ & 0.49 & 0.61 & 1.22 & 0.18 & 0.25 & 0.37 \\
\hline Bremsstrahlung & 0.27 & 0.30 & 0.36 & 0.24 & 0.28 & 0.33 \\
\hline Primary $e^{-}$ & 0.11 & 0.15 & 0.19 & 0.15 & 0.19 & 0.24 \\
\hline Secondary $e^{ \pm}$ & 0.16 & 0.15 & 0.17 & 0.09 & 0.09 & 0.09 \\
\hline$\gamma$-rays $(0.1-100 \mathrm{GeV})$ : & 8.86 & 9.12 & 10.3 & 6.72 & 7.18 & 7.79 \\
\hline$\pi^{0}$-decay & 6.75 & 6.46 & 6.59 & 4.99 & 4.90 & 4.79 \\
\hline Inverse Compton & 1.25 & 1.76 & 2.59 & 1.27 & 1.77 & 2.43 \\
\hline Primary $e^{-}$ & 1.15 & 1.66 & 2.43 & 1.20 & 1.68 & 2.33 \\
\hline Secondary $e^{ \pm}$ & 0.10 & 0.10 & 0.16 & 0.07 & 0.09 & 0.10 \\
\hline Bremsstrahlung & 0.87 & 0.88 & 1.08 & 0.46 & 0.51 & 0.57 \\
\hline Primary $e^{-}$ & 0.51 & 0.58 & 0.74 & 0.33 & 0.39 & 0.45 \\
\hline Secondary $e^{ \pm}$ & 0.36 & 0.30 & 0.34 & 0.13 & 0.12 & 0.12 \\
\hline Radio $(0.001-100 \mathrm{GHz})$ : & 2.07 & 2.76 & 3.72 & 1.48 & 2.03 & 2.50 \\
\hline Primary $e^{-}$ & 1.65 & 2.31 & 3.10 & 1.30 & 1.81 & 2.26 \\
\hline Secondary $e^{ \pm}$ & 0.42 & 0.45 & 0.62 & 0.18 & 0.22 & 0.24 \\
\hline \multicolumn{7}{|l|}{ Conversion efficiencies: } \\
\hline$\gamma$-rays/CR leptons ${ }^{\mathrm{a}}$ & 0.34 & 0.40 & 0.57 & 0.28 & 0.34 & 0.42 \\
\hline Synchrotron/CR leptons ${ }^{\mathrm{b}}$ & 0.17 & 0.19 & 0.22 & 0.13 & 0.16 & 0.17 \\
\hline Lepton calorimetric efficiency ${ }^{c}$ & 0.51 & 0.59 & 0.79 & 0.41 & 0.49 & 0.59 \\
\hline Optical-FIR (0.3-3000 THz): & \multicolumn{6}{|c|}{$24.8 \times 10^{43} \mathrm{erg} \mathrm{s}^{-1}$} \\
\hline Optical $^{\mathrm{d}}$ & \multicolumn{6}{|c|}{$19.6 \times 10^{43} \mathrm{erg} \mathrm{s}^{-1}$} \\
\hline Total $\mathrm{IR}^{\mathrm{e}}$ & \multicolumn{6}{|c|}{$5.2 \times 10^{43} \mathrm{erg} \mathrm{s}^{-1}$} \\
\hline FIR $^{\mathrm{f}}$ & \multicolumn{6}{|c|}{$1.9 \times 10^{43} \mathrm{erg} \mathrm{s}^{-1}$} \\
\hline \multicolumn{7}{|l|}{ Radio-FIR relation: } \\
\hline Synchrotron $(1.4 \mathrm{GHz})^{\mathrm{g}}$ & 1.68 & 2.18 & 2.85 & 1.06 & 1.49 & 1.82 \\
\hline$q_{\mathrm{FIR}}$ & 2.49 & 2.38 & 2.26 & 2.69 & 2.54 & 2.45 \\
\hline
\end{tabular}

Notes.

a (IC+bremsstrahlung)/(total leptons).

b (Synchrotron)/(total leptons).

c (IC+bremsstrahlung+synchrotron)/(total leptons).

d $0.1-8 \mu \mathrm{m} ; 38-3000 \mathrm{THz}$.

e $8-1000 \mu \mathrm{m} ; 0.3-38 \mathrm{THz}$

f $42.5-122.5 \mu \mathrm{m} ; 2.45-7.06 \mathrm{THz}$.

g $10^{28} \mathrm{erg} \mathrm{s}^{-1} \mathrm{~Hz}^{-1}$.

A.W.S. and T.A.P. would like to thank the organizers of the Infrared Emission, Interstellar Medium, and Star Formation Workshop (http://www.mpia-hd.mpg.de/IR10/) and the participants for the many stimulating talks and discussions. We would also like to thank George Helou and Heinz Völk for useful comments. T.A.P. acknowledges support from NASA Grant No. NNX10AE78G. I.V.M. acknowledges support from NASA Grant No. NNX09AC15G.

\section{REFERENCES}

Abdo, A. A., et al. 2009a, Phys. Rev. Lett., 102, 181101 Abdo, A. A., et al. 2009b, ApJ, 703, 1249

Abdo, A. A., et al. 2009c, Phys. Rev. Lett., 103, 251101
Abdo, A. A., et al. 2010a, ApJ, 710, 133

Abdo, A. A., et al. 2010b, Phys. Rev. Lett., 104, 101101

Ando, S., \& Pavlidou, V. 2009, MNRAS, 400, 2122

Cox, D. P. 2005, ARA\&A, 43, 337

de Jong, T., et al. 1985, A\&A, 147, L6

de Nolfo, G. A., et al. 2006, Adv. Space Res., 38, 1558

Diehl, R., et al. 2006, Nature, 439, 45

Dogiel, V. A., et al. 2002, ApJ, 572, 157

Engelmann, J. J., et al. 1990, A\&A, 233, 96

Ferrière, K. M. 2001, Rev. Mod. Phys., 73, 1031

Freudenreich, H. T. 1998, ApJ, 492, 495

Helou, G., et al. 1985, ApJ, 298, 7

Kent, S. M., et al. 1991, ApJ, 378, 131

Lorimer, D. R. 2004, in IAU Symp. 218, Young Neutron Stars and Their Environments, ed. F. Camilo \& B. M. Gaensler (Cambridge: Cambridge Univ. Press), 105 
Moskalenko, I. V., \& Strong, A. W. 1998, ApJ, 493, 694

Moskalenko, I. V., et al. 2002, ApJ, 565, 280

Murphy, E. J., et al. 2006, ApJ, 638, 157

Murphy, E. J., et al. 2008, ApJ, 678, 828

Paladini, R., et al. 2007, A\&A, 465, 839

Porter, T. A., et al. 2008, ApJ, 682, 400

Sironi, L., \& Socrates, A. 2010, ApJ, 710, 891

Socrates, A., et al. 2008, ApJ, 687, 202

Sodroski, T. J., et al. 1997, ApJ, 480, 173
Strong, A. W., \& Moskalenko, I. V. 1998, ApJ, 509, 212

Strong, A. W., et al. 2000, ApJ, 537, 763

Strong, A. W., et al. 2004a, ApJ, 613, 962

Strong, A. W., et al. 2004b, A\&A, 422, L47

Strong, A. W., et al. 2007, Ann. Rev. Nucl. Part. Sci., 57, 285

Thompson, T. A., Quataert, E., \& Waxman, E. 2007, ApJ, 654, 219

Völk, H. J. 1989, A\&A, 218, 67

Yanasak, N. E., et al. 2001, ApJ, 563, 768

Yun, M. S., et al. 2001, ApJ, 554, 803 